\title{
Criterion for analyzing experimental data on eddy diffusion coefficients
}

\author{
M. N. Vlasov and M. C. Kelley \\ School of Electrical and Computer Engineering, Cornell University, Ithaca, NY, USA \\ Correspondence to: M. N. Vlasov (mv75@cornell.edu)
}

Received: 31 May 2013 - Revised: 12 April 2014 - Accepted: 15 April 2014 - Published: 2 June 2014

\begin{abstract}
Problems exist in estimating the eddy heat transport coefficient, $K_{\mathrm{eh}}$, from experimental data. These problems are due to uncertainty in determining the turbulent energy dissipation rate and to the uncertainty of $K_{\text {eh }}$ dependence on the energy dissipation rate. In this paper, a new criterion for estimating the eddy heat transport coefficient is suggested. This criterion is based on the effect of eddy turbulence on the energy budget of the upper mesosphere and lower thermosphere. The calculations show high cooling around and above the $K_{\text {eh }}$ peak for $K_{\text {eh }}$ values inferred from experimental data. The cooling rates are much higher than cooling rates corresponding to the temperature given by the MSIS-E-90 model or to temperatures measured during the experiments. The main contribution to high cooling rates is due to the term with eddy heat conduction, which strongly depends on the $K_{\text {eh }}$ gradient. According to our results, the heating/cooling values below the $K_{\text {eh }}$ peak altitude correspond to the temperature given by the MSIS-E-90 model, but at the peak and above, the cooling rates are larger by a factor of 2-3 than the rates corresponding to the temperatures. This means that the $K_{\text {eh }}$ values in the peak and above may be overestimated. Application of this criterion to the Turbulent Oxygen Mixing Experiment (TOMEX) data shows that eddy diffusions inferred from observing chemical tracers in TOMEX are strongly overestimated.
\end{abstract}

Keywords. Atmospheric composition and structure (middle atmosphere - composition and chemistry) - meteorology and atmospheric dynamics (middle atmosphere dynamics; turbulence)

\section{Introduction}

A number of ground-based and in situ measurement techniques for estimating the eddy diffusion coefficient $K_{\text {ed }}$ or eddy heat transport coefficient $K_{\text {eh }}$ exist. Note that the term "eddy diffusion coefficient" is frequently used instead of "eddy heat transport coefficient" in the literature. Radar measurements of the Doppler spectra width or the absolute strength of backscattered power are used to derive the eddy diffusion coefficients (Hocking, 1987). Using ground-based measurements of the green line emission, the eddy diffusion coefficient has been derived. This method is based on the effect of turbulence on the atomic oxygen responsible for the green line emission. Meteor trail observations are used to estimate the eddy diffusion coefficient (Kelley et al., 2003).

Rocket measurements of neutral and electron density fluctuations are used to infer the eddy diffusion coefficient (Lübken, 1997). The density fluctuation is similar to a passive natural tracer variation induced by turbulence. Another rocket-borne technique uses chemiluminescent clouds as tracers released from rockets (Rees et al., 1972; Roper, 1996). All of these methods have limitations and require some theoretical assumptions. The main assumption is linear dependence of the energy dissipation rate on the product of the eddy diffusion coefficient and the square of the buoyancy frequency. Problems with applying this fairly restrictive assumption were noted many times (for example, Fritts and Luo, 1995; Lübken, 1997; Hocking, 1999). We return to this problem later.

The difference between the eddy diffusion coefficient maximum values given by the experimental data exceeds one order of magnitude. Also, a strong contradiction exists between the experimental coefficients and coefficients used in the modeling. In this case, the additional criterion can be useful. 
Our paper is organized as follows. The main uncertainties in determining the eddy diffusion coefficient from experimental data are discussed in Sect. 2. Analysis of eddy diffusion coefficients $K_{\text {eh }}$ inferred from rocket measurements of density fluctuations and rocket-borne chemical tracer releases is presented in Sects. 3 and 4. This analysis is based on estimating the effect of eddy heat transport (eddy diffusion) on the thermal balance in the mesosphere and lower thermosphere (MLT) using the equation for heating/cooling rates by eddy turbulence. The suggested criterion requires agreement between the cooling rate induced by eddy turbulence and the cooling rate corresponding to the temperature given by the MSIS-E-90 model.

\section{Uncertainty of an eddy diffusion coefficient inferred from experimental data}

The energy dissipation rate, $\varepsilon$, is a key parameter in determining the eddy diffusion coefficient, $K_{\text {ed }}$, from experimental data. Usually, the spectrum of density fluctuations calculated from experimental data is approximated using the theoretical model of Heisenberg (1948) and the inner scale, $l_{0}$, is determined. This parameter is related to the Kolmogorov microscale, $\eta$, through the relation $l_{0}=9.9 \eta$ (Lübken et al., 1993). The Kolmogorov microscale is a rough estimate of the size of the smallest eddies, which can provide the turbulent energy dissipation by viscosity $\nu$. Then the $\varepsilon$ value can be calculated using the formula $\varepsilon=v^{3} \eta^{-4}$. According to this formula, the $\varepsilon$ value strongly depends on the $\eta$ value, which is estimated by a rough approximation. For example, let us estimate the impact of $\eta$ values on the energy dissipation rate using the $l_{0}$ values inferred from the experimental data by Kelley et al. (2003). These values vary from 156 to $222 \mathrm{~m}$, and the $\varepsilon$ value can change from 0.14 to $0.58 \mathrm{~W} \mathrm{~kg}^{-1}$. Using the formula $K_{\mathrm{ed}}=b \varepsilon / \omega_{\mathrm{B}}^{2}$ with $b=0.8$ derived by Weinstock (1978) where $\omega_{\mathrm{B}}$ is the buoyancy frequency, Kelley et al. (2003) estimated the $K_{\text {ed }}$ averaged value to be $500 \mathrm{~m}^{2} \mathrm{~s}^{-1}$. Taking into account the $\varepsilon$ variation estimated above, the $K_{\mathrm{ed}}$ maximum values can vary from 250 to $1000 \mathrm{~m}^{2} \mathrm{~s}^{-1}$, covering all $K_{\text {ed }}$ maximum values measured in the mesosphere and lower thermosphere (Fukao et al., 1994). However, it is difficult to imagine that limited experimental data from observing a few meteor trails (Kelley et al., 2003) could present the whole range of $K_{\text {ed }}$ natural variations. In this case, the accuracy of the microscale estimate can play an important role. Note that the $\eta$ change by $20 \%$ corresponds to the $K_{\text {ed }}$ change by a factor of 2 .

The other uncertainty results from the application of formula $K_{\mathrm{ed}}=0.8 \varepsilon / \omega_{\mathrm{B}}^{2}(*)$ (Weinstock, 1978) and the formula $K_{\mathrm{ed}} \omega_{\mathrm{B}}^{2}(P-R i) / R i=\varepsilon(* *)$ where $P$ and $R i$ are the Prandtl and Richardson numbers, respectively. Equation $(* *)$ is derived assuming a balance between the rate of energy transferred from the mean motion to the fluctuations on one side and the rates of turbulent energy dissipation due to viscosity and buoyancy force on the other side in a steady state (Chandra, 1980; Gordiets et al., 1982). This balance assumes that the fluctuations are stationary, homogeneous, and isotropic. However, these conditions are scarcely probable in the real atmosphere. The Eq. $(*)$ derived by Weinstock (1978) is also for stratified homogeneous turbulence. However, this relation is commonly used to estimate $K_{\text {eh }}$ from $\varepsilon$. There is no evidence of any advantage in using Eqs. $(*)$ or $(* *)$, but the latter has an additional problem with $R i$ determination.

The Prandtl number is equal to 1 for uniform turbulence and $R i=0.44$ for $b=0.81$. The Kelvin-Helmholtz instability requires $R i \leq 0.25$, corresponding to $b \leq 0.3$. Equation $(*)$ with $b=0.3$ was obtained by Lilly et al. (1974). However, Weinstock (1978) assumed that the turbulence produced in regions of dynamic instability $(R i \leq 0.25)$ will be transported by turbulent flux into the regions of larger $R i$, and the $R i$ mean value may be 0.44 . Lübken (1997), using Eq. (*), notes that the derivation of this formula relies on fairly restrictive assumptions concerning the turbulent field. Note that a $b$ value equal to 0.8 is used to estimate the $K_{\text {ed }}$ value in all experimental data. The problem when applying Eqs. $(*)$ and $(* *)$ is considered in detail by Hocking (1999).

The same problem exists in estimating the energy dissipation rate inferred from chemical tracer observations. In this case, formula $\varepsilon=r_{\mathrm{t}}^{2} t^{-3} /(2.4 \alpha)^{1.5}$ (Rees et al., 1972, and references therein) is usually used. Here, $r_{\mathrm{t}}$ is the trail radius as a function of time, $t$, and $\alpha$ is a Kolmogorov constant. The values of this constant vary between 0.5 and 1.5 because the absolute value is unknown (Rees et al., 1972; Weinstock, 1978). In this case, the $\varepsilon$ value can change by a factor of 5 due to the uncertainty of a Kolmogorov constant. Note that Bishop et al. (2004) had to use the $\alpha$ maximum value because the energy dissipation rates inferred from the chemical tracer dynamics were unusually high. We will discuss the results of this experiment in Sect. 4. Thus, the uncertainty of the eddy diffusion coefficient inferred from experimental data is a factor of 3 .

\section{Analysis of eddy diffusion coefficients inferred from rocket measurements of density fluctuations}

Using the equation for the heating/cooling rates of $Q_{\mathrm{ed}}$ induced by eddy turbulence, it is possible to estimate these rates for different eddy heat transport coefficients of $K_{\mathrm{eh}}$. Note that the term "eddy diffusion coefficient" is frequently used instead of $K_{\text {eh }}$. The $Q_{\text {ed }}$ estimates show that cooling takes place around and above the $K_{\text {eh }}$ peak. The suggested criterion is based on comparing the calculated cooling rates with normal cooling rates corresponding to the measured temperatures generalized by the MSIS-E-90 model. The enhancement of the cooling induced by eddy turbulence means that the $K_{\text {eh }}$ values inferred from experimental data are too high. Thus, this criterion facilitates estimating the upper limit 


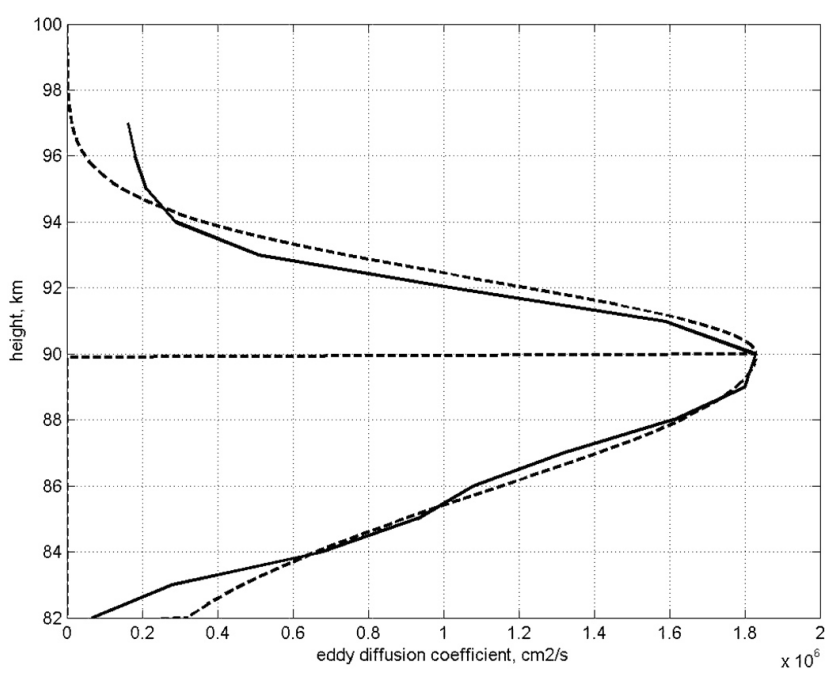

Figure 1. The eddy diffusion coefficient inferred by L97 in polar summer (solid curve) and approximated by Eqs. (2) and (3) (dashed curve). The horizontal dashed line shows the $K_{\text {ed }}$ peak altitude.

of the $K_{\text {eh }}$ values for the $K_{\text {eh }}$ height profile inferred from experimental data. It must be emphasized that the $Q_{\text {ed }}$ value depends on both the $K_{\text {eh }}$ values and the gradient $K_{\text {eh }}$ values. Therefore, both parameters must meet the criterion.

The cooling/heating volume rate corresponding to the eddy diffusion coefficient can be estimated using the equation (Vlasov and Kelley, 2010)

$$
\begin{aligned}
Q_{\mathrm{ed}} & =\frac{\partial}{\partial z}\left[K_{\mathrm{eh}} C_{p} \rho\left(\frac{\partial T}{\partial z}+\frac{g}{C_{p}}\right)\right] \\
& +K_{\mathrm{eh}} \rho \frac{g}{T b}\left(\frac{\partial T}{\partial z}+\frac{g}{C_{p}}\right),
\end{aligned}
$$

where $Q_{\text {ed }}$ is given in $\mathrm{erg} \mathrm{cm}^{-3} \mathrm{~s}^{-1}, \rho$ is the density, $C_{p}$ is the heat capacity at constant pressure, $T$ is the temperature, and $g$ is the gravitational acceleration.

Note that it is usually assumed that the eddy diffusion coefficient, $K_{\text {ed }}$, is equal to the eddy heat transport coefficient, $K_{\text {eh. }}$. The eddy diffusion coefficient inferred by Lübken (1997, hereafter referred to as L97) from measurements of the turbulent energy dissipation rate in the summer polar mesosphere can be approximated by formulas suggested by Shimazaki (1971):

$$
\begin{aligned}
& K_{\mathrm{eh}}=K_{\mathrm{eh}}^{0} \exp \left[S_{1}\left(z-z_{m}\right)\right]+\left(K_{\mathrm{eh}}^{\mathrm{m}}-K_{\mathrm{eh}}^{0}\right) \\
& \quad \exp \left[-S_{2}\left(z-z_{m}\right)^{2}\right] z<z_{m}, \\
& K_{\mathrm{eh}}=K_{\mathrm{eh}}^{\mathrm{m}} \exp \left[-S_{3}\left(z-z_{m}\right)^{2}\right] z>z_{m},
\end{aligned}
$$

where $K_{\mathrm{eh}}^{\mathrm{m}}=1.83 \times 10^{6} \mathrm{~cm}^{2} \mathrm{~s}^{-1}$ is the maximum of these coefficients, $K_{\mathrm{eh}}^{0}=7 \times 10^{5} \mathrm{~cm}^{2} \mathrm{~s}^{-1}$ is the value at $83 \mathrm{~km}$

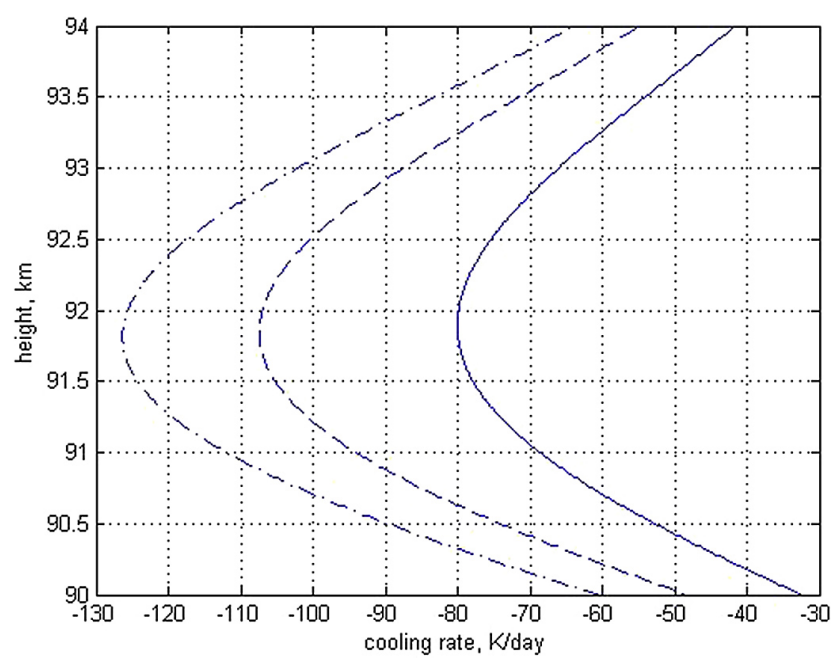

Figure 2. Cooling rates calculated with the eddy heat transport coefficient shown in Fig. 1 with temperature gradient $G=5 \mathrm{~K} \mathrm{~km}^{-1}$ (dotted-dashed curve) corresponding to the temperature height profile shown in Fig. $3, G=3 \mathrm{~K} \mathrm{~km}^{-1}$ (dashed curve), and $G=0$ (solid curve).

according to L97, and $z_{m}=90 \mathrm{~km}, S_{1}=0.05 \mathrm{~km}^{-1}, S_{2}=$ $0.03 \mathrm{~km}^{-2}$, and $S_{3}=0.1 \mathrm{~km}^{-2}$ are free parameters. As seen in Fig. 1, these formulas provide an excellent approximation of the eddy diffusion coefficient presented in Table 3 in L97. Using Eq. (1) with this approximation, it is possible to calculate the cooling/heating rates induced by eddy turbulence. The height profiles of these rates are shown in Fig. 2. Strong cooling takes place above the eddy diffusion coefficient peak and depends on the temperature gradient. The temperature height profile given by the MSIS-E-90 model for conditions corresponding to measurements of L97 is shown in Fig. 3. According to L97, the eddy diffusion coefficient peak is in the mesopause at $90 \mathrm{~km}$. The value of this coefficient is determined using the formula

$K_{\text {ed }}=0.8 \varepsilon / \omega_{\mathrm{B}}^{2}$,

where $\omega_{\mathrm{B}}$ is the buoyancy frequency given by the formula

$\omega_{\mathrm{B}}^{2}=\frac{g}{T}\left(\frac{\partial T}{\partial z}+\frac{g}{C_{p}}\right)$.

Using Eqs. (4) and (5), the $\varepsilon$ and $K_{\text {ed }}$ values given in Table 3 in $\mathrm{L} 97$ and $\mathrm{d} T / \mathrm{d} z=0$, it is possible to estimate the temperature of $139.7 \mathrm{~K}$ at $90 \mathrm{~km}$ in the mesopause. This temperature is in excellent agreement with the temperature in the mesopause shown in Fig. 3, but the mesopause altitude given by the MSIS-E-90 model (Hedin, 1991) is $2 \mathrm{~km}$ lower than the altitude given by L97. This means that we can use the temperature height profile given by MSIS-E-90, shifted up by $2 \mathrm{~km}$. In this case, the temperature gradient is very small within the height range of 90-94 km, and the maximum cooling rate calculated by Eq. (1) is $-80 \mathrm{Kday}^{-1}$, according to 


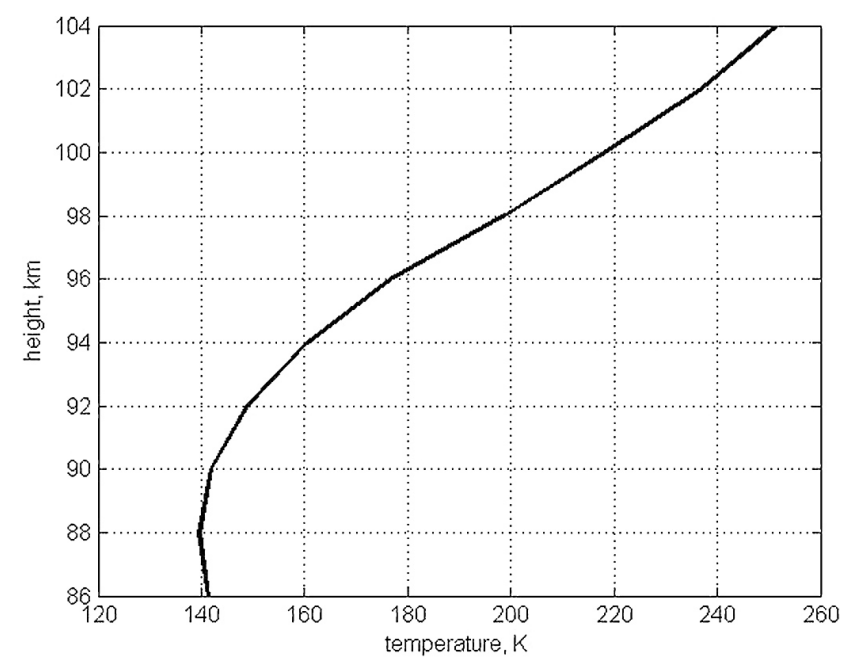

Figure 3. Temperature height profile given by the MSIS-E- 90 model under conditions corresponding to the eddy diffusion coefficient inferred by L97 in polar summer and shown in Fig. 1.

Fig. 2. By comparing these cooling rates with the cooling rates shown in Fig. 10 corresponding to measured temperatures generalized by the MSIS-E-90 model and the temperature used by $\mathrm{L} 97$, we see that the $K_{\text {eh }}$ value does not correspond to the atmospheric conditions. Other heating processes occurring in the MLT cannot compensate for these very high cooling rates. Note that this cooling strongly depends on the $K_{\text {ed }}$ gradient above the $K_{\text {ed }}$ peak, as seen in Fig. 4 . The cooling decreases with decreases in the $K_{\text {ed }}$ gradient. However, in this case, the turbopause altitude significantly increases (see Fig. 5).

In any case, strong cooling occurs above and below the $K_{\text {ed }}$ peak (see Fig. 4). This result contradicts one of the main results of L97 concerning strong heating by eddy turbulence in the summer polar mesopause, meaning that a serious problem exists with the eddy diffusion coefficient estimation method used by L97.

As seen in Fig. 4, the altitude of the maximum heating corresponding to this coefficient is $5 \mathrm{~km}$ lower than the $K_{\text {ed }}$ peak altitude. Note that the maximum heating rate measured by L97 is $13.5 \mathrm{~K} \mathrm{day}^{-1}$, very close to the maximum heating rate of $17 \mathrm{~K} \mathrm{day}^{-1}$ shown in Fig. 4 . This means that the $K_{\text {ed }}$ maximum value estimated by $\mathrm{L} 97$ corresponds not to the altitude of the peak eddy diffusion coefficient in L97, but to the $K_{\text {ed }}$ value at altitudes below $5 \mathrm{~km}$, the $K_{\text {ed }}$ peak.

Considering Eq. (1) in detail, it is possible to show that cooling is in the $K_{\text {eh }}$ peak. Equation (1) for $Q_{\text {ed }}$ in units of $\mathrm{Kday}^{-1}$ for $\mathrm{d} T / \mathrm{d} z=0$ and $z \leq z_{m}$ can be written as

$$
\begin{aligned}
Q_{\mathrm{ed}}^{K} & =p\left(S_{1}-\frac{1}{H}+\frac{g}{T c C_{p}}\right) K_{\mathrm{eh}}^{0} \exp \left[S_{1}\left(z-z_{m}\right)\right] \\
& -p\left[2 S_{2}\left(z-z_{m}\right)+\frac{1}{H}-\frac{g}{T c C_{p}}\right]\left(K_{\mathrm{eh}}^{\mathrm{m}}-K_{\mathrm{eh}}^{0}\right)
\end{aligned}
$$

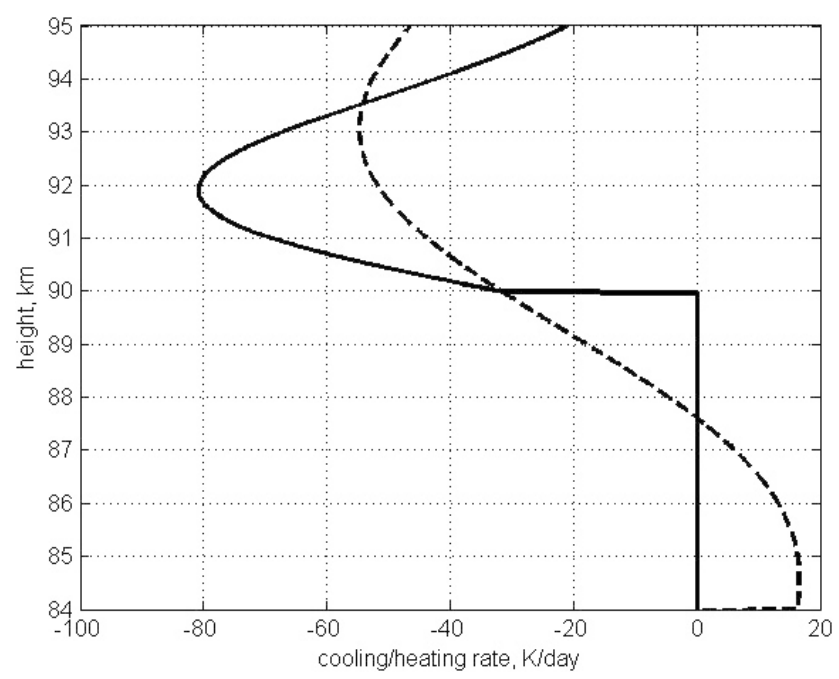

Figure 4. Height profiles of the cooling rates calculated by Eq. (1) with the eddy heat transport coefficient inferred by L97 in summer (solid curve) and $S_{3}=S_{2}=0.03 \mathrm{~km}^{-2}$ (dashed curve), $\mathrm{d} T / \mathrm{d} z=0$, $K_{\mathrm{eh}}^{0}=0$, and $S_{1}=0$. The horizontal solid line shows the altitude of the $K_{\text {eh }}^{\mathrm{m}}$ peak, and the vertical solid line shows the boundary between cooling and heating.

$$
\exp \left[-S_{2}\left(z-z_{m}\right)^{2}\right]
$$

where $p=g \tau_{d} / C_{v}$. The $Q_{\text {ed }}$ value is negative at the $K_{\text {eh }}$ peak altitude because the scale height $H<8 \mathrm{~km}$ in the MLT and $1 / H>\left(S_{1}+g / T c C_{p}\right)$. Cooling above the $K_{\text {eh }}$ peak altitude is due to the $K_{\text {eh }}$ negative gradient.

We suggest that the $K_{\mathrm{eh}}^{\mathrm{m}}$ value can be estimated using the thermal balance equation

$$
\begin{aligned}
& K_{\mathrm{eh}} C_{p} \frac{\partial^{2} T}{\partial z^{2}}+C_{p}\left(\frac{\partial K_{\mathrm{eh}}}{\partial z}+\frac{K_{e}}{\rho} \frac{\partial \rho}{\partial z}\right) \frac{\partial T}{\partial z} \\
& \quad+g\left(\frac{\partial K_{\mathrm{eh}}}{\partial z}+\frac{K_{\mathrm{eh}}}{\rho} \frac{\partial \rho}{\partial z}\right)+\varepsilon+q-L=0,
\end{aligned}
$$

which includes the first term of Eq. (1) divided by mass density $\rho$, heating due to the energy dissipation of gravity waves, $\varepsilon$, chemical heating and heating by ultraviolet solar radiation, $q$, and cooling by $\mathrm{CO}_{2}$ and $\mathrm{O}$ infrared radiation, $L$.

Note that $(1 / \rho) \partial \rho / \partial / z=-1 / H$ for $\partial T / \partial z=0$ and $(1 / \rho) \partial \rho / \partial / z=-(\alpha+m g / \kappa) /\left(T_{0}+\alpha z\right)$, where $m$ is the mass, for $\partial T / \partial z=\alpha$. According to the conditions in L97, the $K_{\text {ed }}$ peak is in the mesopause $(\partial T / \partial z=0)$ and Eq. (7) can be simplified to the relation

$K_{\mathrm{eh}}^{\mathrm{m}} g / H=q+\varepsilon-L$.

Using this relation with the $\varepsilon$ value given in Table 3 in L97 at the $K_{\text {ed }}$ peak altitude and $(q-L) \leq 10 \mathrm{Kday}^{-1}, K_{\text {eh }}^{\mathrm{m}}$ is found to be $\leq 1.1 \times 10^{6} \mathrm{~cm}^{2} \mathrm{~s}^{-1}$. This value is significantly less than $K_{\mathrm{eh}}^{\mathrm{m}}=1.83 \times 10^{6} \mathrm{~cm}^{2} \mathrm{~s}^{-1}$ as estimated by L97, but the maximum cooling with this lower value is $-45 \mathrm{~K}$ day $^{-1}$ 


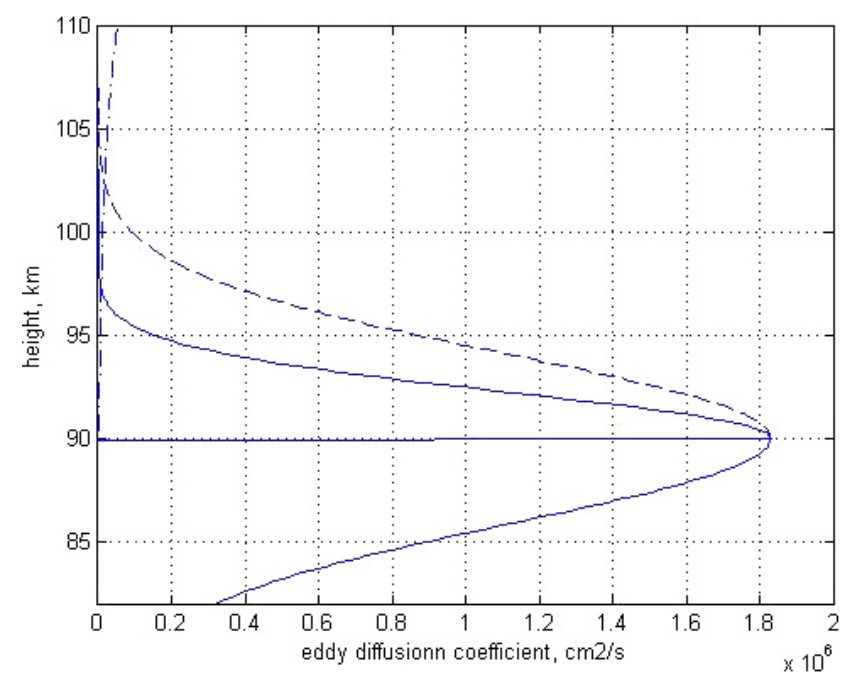

Figure 5. The eddy diffusion coefficient (L97) (solid curve) with $S_{3}=0.03 \mathrm{~km}^{-1}$ (dashed curve) and the molecular diffusion coefficient (dotted-dashed line). The horizontal line shows the altitude of the $K_{\text {eh }}$ peak.

due to the large $S_{3}$ value corresponding to the $K_{\text {ed }}$ height profile given by L97. The $S_{3}$ value should decrease by a factor of 10 to maintain thermal balance at altitudes above the $K_{\text {ed }}$ peak. However, in this case, the turbopause altitude can be too high.

Thus, in this case, the cooling induced by the eddy diffusion measured by Lübken is very large, resulting in a $P$ value larger than 2 and localized turbulence.

\section{Analysis of eddy diffusion coefficients inferred from a rocket-borne chemical tracer experiment}

We now consider estimates of the eddy diffusion coefficient inferred from observing chemical tracers during a rocketborne experiment (Bishop et al., 2004, hereafter referred to as B04). The energy dissipation rate and the eddy diffusion coefficients calculated by Eq. (4) are given in Tables 1 and 2 in B04. Height profiles of the eddy diffusion coefficients given in Table 2 are shown in Fig. 6. These profiles have two peaks at 87 and $102 \mathrm{~km}$ altitude. The steep, negative temperature gradients were observed in these peaks, and the temperatures can be described by the linear function $T(z)=T_{0}-\alpha z$, as seen in Fig. 2 in Hecht et al. (2004). Using Eq. (7) and $(1 / \rho) \partial \rho / \partial / z=-(\alpha+m g / \kappa) /\left(T_{0}+\alpha z\right)$ for $\partial T / \partial z=-\alpha, \partial^{2} T / \partial z^{2}=0$ and $\partial K_{\text {eh }} / \partial z=0$ in the $K_{\text {eh }}$ peak, it is possible to obtain the formula

$K_{\mathrm{eh}}^{\mathrm{m}}=\frac{(\varepsilon+q-L) T}{C_{p}(P-\alpha)\left(g / C_{p}-\alpha\right)}$,

where $P=T / H$. Equation (4) is used by B04 to estimate the $K_{\mathrm{eh}}^{\mathrm{m}}$ value. Using Eq. (9) and the $\varepsilon$ and $K_{\mathrm{eh}}^{\mathrm{m}}$ values at $87 \mathrm{~km}$,

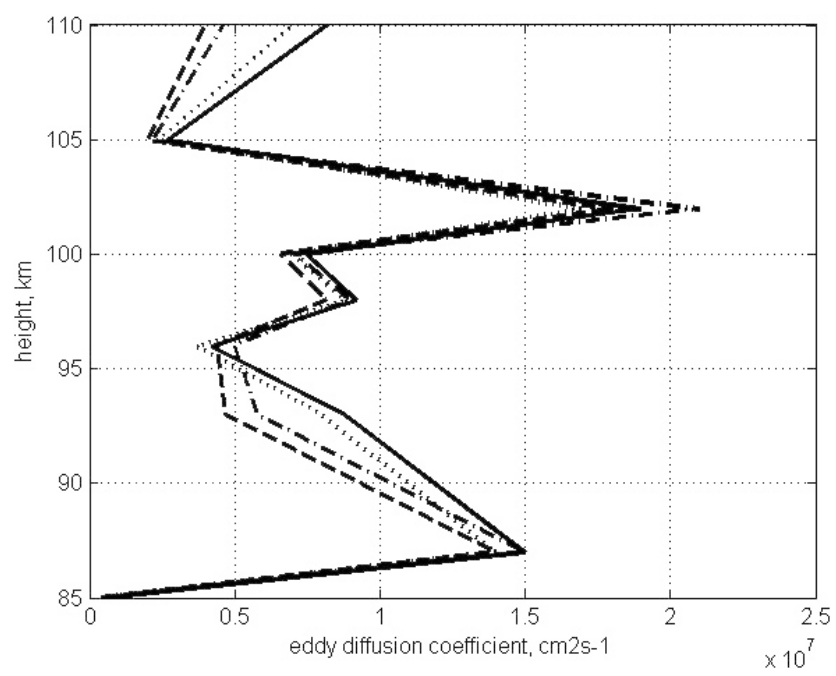

Figure 6. Height profiles of eddy diffusion coefficients inferred from the rocket-borne chemical tracer experiment given in Table 2 for methods 1-4 (Bishop et al., 2004) are shown by solid, dasheddotted, dotted, and dashed lines, respectively.

as given in Tables 1 and 2 in B04, $\omega_{\mathrm{B}}^{2}$ can be found. Then the temperature gradient can be estimated to be $-8 \mathrm{~K} \mathrm{~km}^{-1}$ within the height range of 86 to $95 \mathrm{~km}$ by using the temperature height profile shown in Fig. 2 in Hecht et al. (2004), and $K_{\text {eh }}^{\mathrm{m}}=7.7 \times 10^{6} \mathrm{~cm}^{2} \mathrm{~s}^{-1}$ can be found. This value is less by a factor of about 2 than the $K_{\mathrm{eh}}^{\mathrm{m}}=1.5 \times 10^{7} \mathrm{~cm}^{2} \mathrm{~s}^{-1}$ value estimated by Bishop et al. (2004). This difference shows that a problem exists with the application of Eq. (4). We discuss this problem later. The cooling rates calculated with $K_{\mathrm{eh}}^{\mathrm{m}}=1.5 \times 10^{7} \mathrm{~cm}^{2} \mathrm{~s}^{-1}$ and the $K_{\mathrm{eh}}$ approximation by Eq. (3) within the altitude range of $87-96 \mathrm{~km}$ are shown in Fig. 7. This cooling would be in agreement with the strong negative temperature gradient estimated above if it did not contradict the very high temperature measured just below the $K_{\text {eh }}$ peak (see Hecht et al., 2004). A very strong source of heating is necessary to increase the temperature by $35 \mathrm{~K}$, higher than normal temperatures at $85 \mathrm{~km}$ altitude. Note that the eddy turbulence can heat the atmosphere above the $K_{\text {eh }}$ peak if convective instability $\left(-\partial T / \partial z>g / C_{p}\right)$ occurs. It is possible to assume that this instability took place before the observations.

We now consider the eddy diffusion coefficients inferred by Bishop et al. (2004) at $102-105 \mathrm{~km}$ altitude and shown in Fig. 6 with $K_{\mathrm{eh}}^{\mathrm{m}}=2.1 \times 10^{7} \mathrm{~cm}^{2} \mathrm{~s}^{-1}$. The height distribution approximation of this $K_{\text {eh }}$ by Eq. (3) and the linear dependence are shown in Fig. 8. Using the approach described above, it is possible to estimate the temperature gradient used in Eq. (4) by Bishop et al. (2004) for their estimates of the $K_{\text {eh }}$ values. The cooling rate height profile calculated with the $K_{\text {eh }}$ approximation by Eq. (3) is shown in Fig. 9. These cooling rates are much higher than the normal cooling rates shown in Fig. 10. This cooling is very large and cannot be 


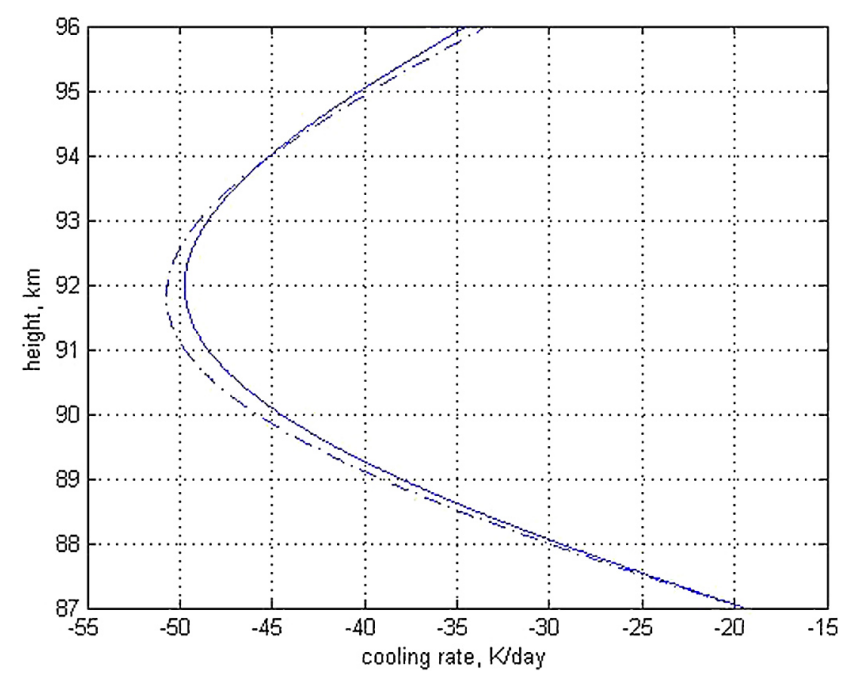

Figure 7. Height profiles of cooling rates corresponding to the $K_{\mathrm{eh}}^{\mathrm{m}}$ value estimated by Bishop et al. (2004) at $87 \mathrm{~km}$ and the exponential approximation of the $K_{\text {eh }}$ height profile above the peak with the $K_{\text {eh }}$ gradient, $S_{3}$, equal to $0.015 \mathrm{~km}^{-2}$ (solid curve) and $0.016 \mathrm{~km}^{-2}$ (dashed-dotted curve).

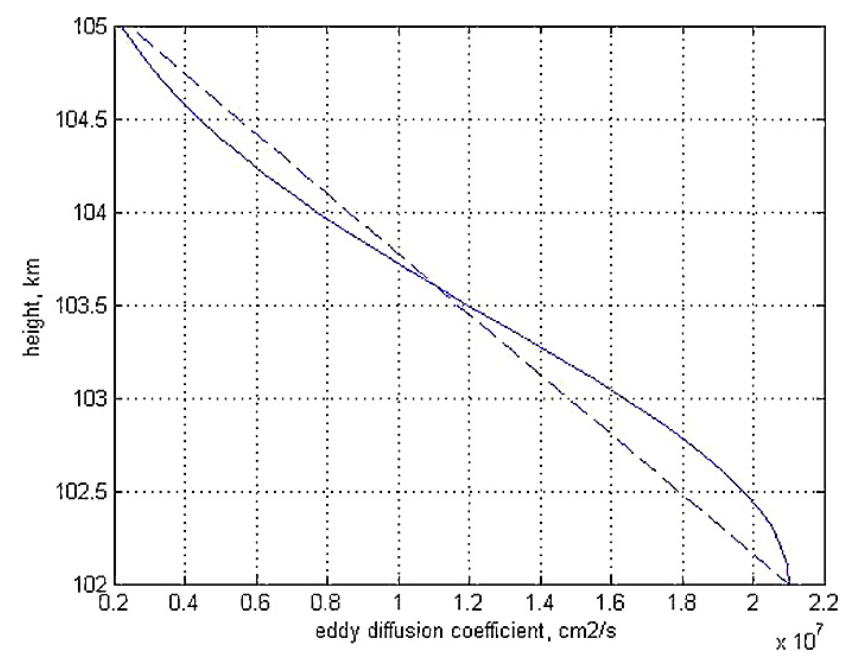

Figure 8. The approximation by Eq. (3) (solid curve) and the linear approximation (dashed line) of the eddy diffusion coefficients given in Table 2 in Bishop et al. (2004) within the altitude range of 102$105 \mathrm{~km}$.

realistic. The cooling calculated with the linear approximation is a little smaller but is also too high.

Finally, our analysis shows that the eddy diffusion coefficients inferred from observing chemical tracers are overestimated. Note that the coefficients used by TIME-GCM to provide a better fit to the Turbulent Oxygen Mixing Experiment (TOMEX) photometer data do not exceed $3 \times 10^{6} \mathrm{~cm}^{2} \mathrm{~s}^{-1}$.

The results presented above mean that a contradiction exists between Eq. (4), commonly used to estimate the eddy diffusion coefficient from experimental data, and the real eddy

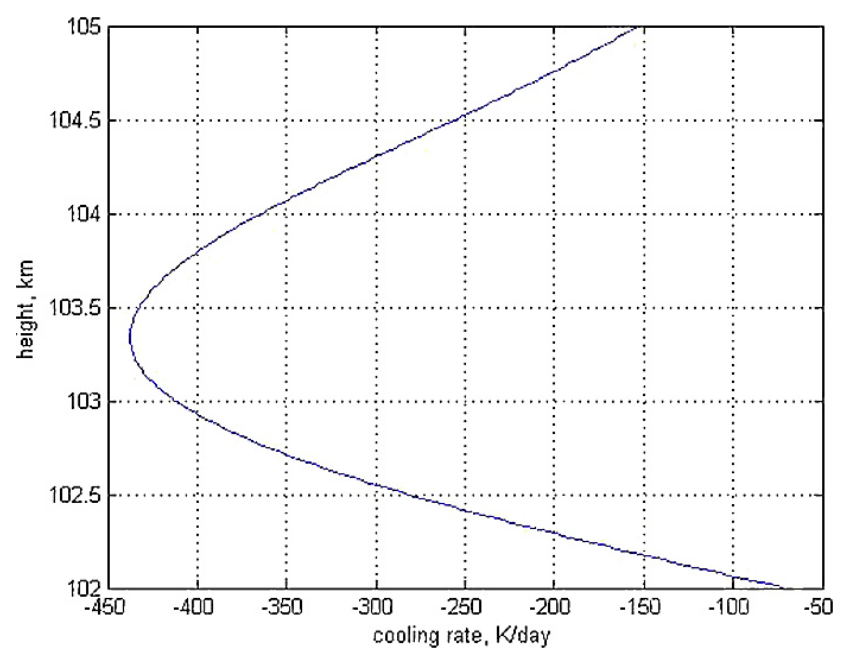

Figure 9. Cooling rates calculated with the $K_{\text {eh }}$ approximation shown in Fig. 8 (solid curve).

turbulence. Hocking (1999) discussed this problem in detail and concluded that the application of this formula depends on the eddy scales. However, it seems to us that Hocking and the other authors use the term "eddy diffusion" in extended interpretations, including diffusion with scales compared to atmospheric scales. We believe that eddy diffusion is the process that meets the main diffusion criterion: eddy scales are much less than the density gradient scale. This diffusion can only be used in diffusion equations and can induce small fluctuations of mass density but cannot induce mass density transport. Large-scale diffusion can be considered as the mass transport (advection), which can induce a change of total density and can be described by the momentum equation.

\section{Conclusions}

Deriving the eddy heat transport (eddy diffusion) coefficient from experimental data is a very complicated problem. There are two main uncertainties: (i) estimating the turbulent energy dissipation rate and (ii) determining the dependence of the heat transport coefficient on the energy dissipation rate. The $K_{\text {eh }}$ value can be underestimated or overestimated by a factor of 2-4 due to these uncertainties. Thus, an independent criterion for the $K_{\text {eh }}$ value estimate can be useful.

One of the main features of eddy diffusion is that it always cools atmospheric gas at altitudes around and above the eddy diffusion coefficient peak. Our analysis shows that the cooling rates calculated by the energy rate equation with eddy heat transport coefficients inferred from experimental data can be used as the criterion for analyzing these experimental data. By comparing these cooling rates with cooling rates corresponding to measured temperatures generalized by the MSIS-E-90 model, the $K_{\text {eh }}$ value's correspondence with 


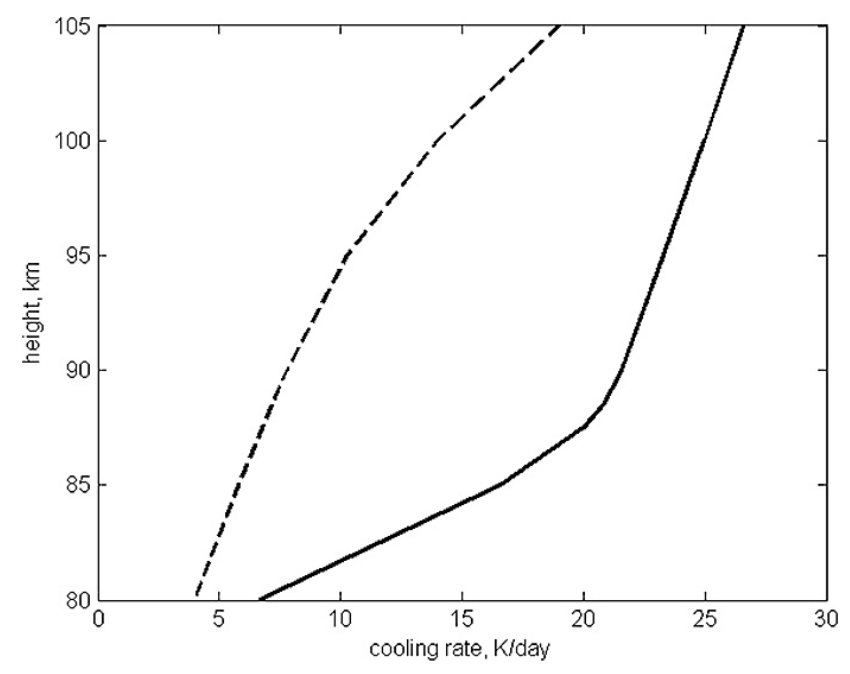

Figure 10. Normal cooling rates corresponding to MSIS-E-90 data for the conditions in B04 (dashed curve) and L97 (solid curve).

or contradiction to the atmospheric temperatures can be obtained.

The simplified formulas based on the energy rate equation can be used to estimate the eddy diffusion coefficient from the energy dissipation rate given by the experimental data. These formulas give the eddy diffusion coefficients, which are significantly less than the coefficients estimated by the commonly used Eq. (4).

Applying this criterion to the eddy diffusion coefficient inferred from the rocket experimental data on density fluctuations, L97 shows that the eddy diffusion coefficients inferred from density fluctuations (L97) at the $K_{\text {ed }}$ peak altitude and below meet our criterion. However, the cooling rate above the peak is too large due to the very steep gradient of this coefficient. The cooling rates calculated with the coefficients, estimated using chemical tracers in TOMEX (Bishop et al., 2004), are very high due to the large $K_{\text {eh }}$ value in the peak and the very steep gradient above the peak. These coefficients are significantly overestimated. The main problem with this technique is that the non-turbulent effects can influence the tracer dynamic. For example, the molecular diffusion coefficient $D_{\mathrm{m}}$ estimated by B04 is $1.6 \times 10^{7} \mathrm{~cm}^{2} \mathrm{~s}^{-1}$ at $116 \mathrm{~km}$ and $2.1 \times 10^{7} \mathrm{~cm}^{2} \mathrm{~s}^{-1}$ and $2.6 \times 10^{7} \mathrm{~cm}^{2} \mathrm{~s}^{-1}$ at $128 \mathrm{~km}$. As is well known, the molecular diffusion coefficient is proportional to the reciprocal of the total density. In this case, the $D_{\mathrm{m}}$ value should increase by a factor of 3 within the altitude range of $116-128 \mathrm{~km}$. Also, the $D_{\mathrm{m}}$ value increases with increasing temperature. The estimated total $D_{\mathrm{m}}$ increases by a factor of 3.5, a factor of 2 larger than the $D_{\mathrm{m}}$ increase estimated in B04. We believe that the suggested criterion can encourage the B04 authors to reconsider their results.
Acknowledgements. Work at Cornell was supported by the National Science Foundation under grant ATM-0551107.

Topical Editor R. Neale thanks two anonymous referees for their help in evaluating this paper.

\section{References}

Bishop, R. L., Larsen, M. F., Hecht, J. H., Liu, A. Z., and Gardner, C. S.: TOMEX: Mesospheric and lower thermospheric diffusivity and instability layers, J. Geophys. Res., 109, D02S03, doi:10.1029/2002JD003079, 2004.

Chandra, S.: Energetics and thermal structure of the middle atmosphere, Planet. Space Sci., 28, 585-593, 1980.

Fritts, D. C. and Luo, Z.: Dynamical and radiative forcing of the summer mesopause circulation and thermal structure, J. Geophys. Res., 100, 3119-3128, 1995.

Fukao, S., Yamanaka, M. D., Ao, N., Hocking, W. K., Sato, T., Yamamoto, M., Nakamura, T., Tsuda, T., and Kato, S.: Seasonal variability of vertical eddy diffusivity in the middle atmosphere, J. Geophys. Res., 99, 18973-18987, 1994.

Gordiets, B. F., Kulikov, Yu. N., Markov, M. N., and Marov, M. Ya.: Numerical modeling of the thermospheric heat budget, J. Geophys. Res., 87, 4504-4514, 1982.

Hecht, J. H., Liu, A. Z., Walterscheid, R. L., Roble, R. G., Larsen, M. F., and Clemmons, J. H.: Airglow emissions and oxygen mixing ratios from the photometer experiment on the Turbulent Oxygen Mixing Experiment (TOMEX), J. Geophys. Res., 109, D02S05, doi:10.1029/2002JD003035, 2004.

Hedin, A. E.: Extension of the MSIS thermosphere model into the middle and lower atmosphere, J. Geophys. Res., 96, 1159-1172, 1991.

Heisenberg, W.: Zur statistischen Theorie der Turbulenz, Z. Phys., 124, 628-657, 1948.

Hocking, W. K.: Turbulence in the region 80-120 km, Adv. Space Res., 7, 171-181, 1987.

Hocking, W. K.: The dynamical parameters of turbulence theory as apply to middle atmosphere studies, Earth Planets Space, 51, 525-541, 1999.

Kelley, M. C., Kruschwitz, C. A., Gardner, C. S., Drummond, J. D., and Kane, T. J.: Mesospheric turbulence measurements from persistent Leonid meteor train observations, J. Geophys. Res., 108, 8454, doi:10.1029/2002JD002392, 2003.

Lilly, D. K., Wasco, D. E., and Adelfang, S. I.: Stratospheric mixing estimated from high-altitude turbulence measurements, J. Appl. Meteorol., 13, 488-493, 1974.

Lübken, F. J.: Seasonal variation of turbulent energy dissipation rates at high latitudes as determined by in situ measurements of neutral density fluctuations, J. Geophys. Res., 102, 1344113456, 1997.

Lübken, F. J., Hillert, W., Lehmacher, G., and von Zahn, U.: Experiments revealing small impact of turbulence on the energy budget of the mesosphere and lower thermosphere, J. Geophys. Res., 98, 20369-20384, 1993.

Rees, D., Roper, R. G., Lloyd, K. H., and Low, C. H.: Determination of the structure of the atmosphere between 90 and $250 \mathrm{~km}$ by means of contaminant releases at Woomera, May 1968, Philos. T. Roy. Soc. London A, 271, 631-663, 1972. 
Roper, R. G.: Rocket vapor trail releases revisited: Turbulence and the scale of gravity waves: Implications for the imaging Doppler interferometry/incoherent scatter radar controversy, J. Geophys. Res., 101, 7013-7017, 1996.

Shimazaki, T.: Effective eddy diffusion coefficient and atmospheric composition in the lower thermosphere, J. Atmos. Terr. Phys., 33, 1383-1401, 1971.
Vlasov, M. N. and Kelley, M. C.: Estimates of eddy turbulence consistent with seasonal variations of atomic oxygen and its possible role in the seasonal cycle of mesopause temperature, Ann. Geophys., 28, 2103-2110, doi:10.5194/angeo-28-2103-2010, 2010.

Weinstock, J.: Vertical turbulent diffusion in a stably stratified fluid, J. Atmos. Sci., 35, 1022-1027, 1978. 